recht durch Begründung besonderer Verhaltens- und Kostentragungspflichten de lege ferenda sind insbesondere die damit notwendig verbundenen Eingriffe in grundrechtsgeschützte Positionen der betroffenen Unternehmen zu bedenken. Diese können grundsätzlich durch gegenläufige Belange des Schutzes der menschlichen Gesundheit und der Umwelt gerechtfertigt werden. Damit werden komplexe Verhältnismäßigkeitsprüfungen in Rechtssetzung und Rechtsanwendung erforderlich, die nicht pauschal antizipiert werden können, sondern unter Berücksichtigung des konkret nachweislichen oder belastbar prognostizierten Gefahren- und Risikopotenzials der verschiedenen Stoffe und Stoffgruppen für die konkret in Erwägung gezogenen einzelnen erweiterten Rechtspflichten der betroffenen Hersteller vorzunehmen sind.

(5) Eine erweiterte Hersteller- und Produktverantwortung im Abwasserrecht ist kompetenzrechtlich sowohl auf unionsrechtlicher als auch auf bundesrechtlicher Ebene möglich. Insbesondere mit Rücksicht auf den grenzüberschreitenden Wettbewerb erscheint dabei eine europäische Lösung vorzugswürdig. Dabei würde sich in rechtssetzungstechnischer Hinsicht eine entsprechende Regelung nach dem Vorbild des europäischen Abfallrechts als Ergänzung der kommunalen Abwasserrichtlinie anbieten.

Open Access. Dieser Artikel wird unter der Creative Commons Namensnennung 4.0 International Lizenz veröffentlicht, welche die Nutzung, Vervielfältigung, Bearbeitung, Verbreitung und Wiedergabe in jeglichem Medium und Format erlaubt, sofern Sie den/die ursprünglichen Autor(en) und die Quelle ordnungsgemäß nennen, einen Link zur Creative Commons Lizenz beifügen und angeben, ob Änderungen vorgenommen wurden.

Die in diesem Artikel enthaltenen Bilder und sonstiges Drittmaterial unterliegen ebenfalls der genannten Creative Commons Lizenz, sofern sich aus der Abbildungslegende nichts anderes ergibt. Sofern das betreffende Material nicht unter der genannten Creative Commons Lizenz steht und die betreffende Handlung nicht nach gesetzlichen Vorschriften erlaubt ist, ist für die oben aufgeführten Weiterverwendungen des Materials die Einwilligung des jeweiligen Rechteinhabers einzuholen.

Weitere Details zur Lizenz entnehmen Sie bitte der Lizenzinformation auf http://creativecommons.org/licenses/by/4.0/deed.de.

Open Access funding enabled and organized by Projekt DEAL.

\title{
https://doi.org/10.1007/s10357-020-3785-z
}

\section{Das Vorsorgeprinzip beim Ausbau von G5}

\section{Hans-Jürgen Müggenborg}

\section{(c) Springer-Verlag GmbH Deutschland, ein Teil von Springer Nature 2021}

Der Ausbau des neuen Mobilfunkstandards 5G steht in den Startlöchern und hat mancherorts bereits begonnen. $\mathrm{Zu}$ fragen ist, ob der weitgehend ungeregelte Ausbau dieser neuen Technologie vor dem Hintergrund zahlreicher Untersuchungen, die auf ein gesundheitliches Gefährdungspotenzial der Technik hinweisen, überhaupt zulässig ist.

\section{Problemaufriss}

Hohe Übertragungsraten und schnelles Internet haben unbestreitbar Vorteile. Deshalb sehen manche Branchen dem zügigen Ausbau des neuen Mobilfunknetzes $5 \mathrm{G}$, die auch als Schlüsseltechnologie für den digitalen Wandel bezeichnet wird, mit hohen Erwartungen entgegen. Autonomes Fahren, eHealth ${ }^{1}$ oder das Internet-of-Things etwa würden ohne $5 \mathrm{G}$ nicht funktionieren.

Auf der anderen Seite mehren sich Stimmen, die vor den negativen Folgen von $5 \mathrm{G}$ warnen. ${ }^{2}$ Befürchtet werden gesundheitliche Schäden durch die Funkstrahlung, angefangen von Schlafstörungen bis hin zu Krebs. Auf der anderen Seite wird darauf hingewiesen, dass es weltweit keine Studie gibt, die die Unschädlichkeit der 5G-Mikrowellen-Funktechnologie belegt, wohl aber zahlreiche Studien, die vor den gesundheitlichen Folgen warnen. Namhafte Ärzte haben in einem offenen Brief vom 6.4.2020 an Bundeskanzlerin Angela Merkel auf eine Schwächung des Immunsystems durch 5G-Strahlung hingewiesen. ${ }^{3}$ Es gibt ferner den vom IFUR - Privates Institut für Urfeldforschung von Wolfgang Kühl am 24.4.2020 veröffent-

Prof. Dr. jur. Hans-Jürgen Müggenborg,

Rechtsanwalt und Fachanwalt für Verwaltungsrecht,

Aachen, Deutschland lichten Verdacht, dass die hohen 60 GHZ-Frequenzen des $5 \mathrm{G}-\mathrm{B} a n d e s$ zu einer physikalischen Veränderung von Sauerstoffmolekülen führen, so dass der Sauerstoff vom Blut nicht mehr problemlos transportiert werden kann. ${ }^{4} \mathrm{Zu}-$ dem wird der Energiehunger der Welt bei einem flächendeckenden 5G-Ausbau rapide steigen. Der dazu nötige Strombedarf wird, solange die Energie nicht zu $100 \%$ aus erneuerbaren Energien gewonnen wird ${ }^{5}$, nicht ohne weitere Belastungen für die Umwelt gedeckt werden können.

1) Siehe dazu das eHealth-Gesetz: Gesetz für sichere digitale Kommunikation und Anwendungen im Gesundheitswesen sowie Änderung weiterer Gesetze v. 21.12.2015, BGB1. I S. 2408.

2) Budzinski, NuR 2008, $535 \mathrm{ff}$. ders., NVwZ 2010, $1205 \mathrm{ff}$; ders., NVwZ 2012, $547 \mathrm{ff}$;; ders., NVwZ 2012, $1593 \mathrm{ff.}$; ders., NVwZ 2013, $404 \mathrm{ff}$; ders., NuR 2013, $613 \mathrm{ff}$; ders., NVwZ 2013, $988 \mathrm{ff}$.; ders., NuR 2017, 757 ff.; ders., WuW 2019, 508 ff.; Budzinski/Hut-

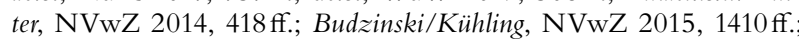
Wahlfels, NVwZ 2013, 653ff.; Appel/Bulla, DVBl. 2008, $1277 \mathrm{ff}$.; Gratzel/Kniep, WuM 2009, 383 ff.; Kniep, ZMR 2009, 510 ff.; Gehrken/Kahle/Mechel, ZUR 2006, 72ff.; Koch, NVwZ 2013, $251 \mathrm{ff}$.

3) Offener Brief an die Bundesregierung, Stand 17.11.2020, abrufbar unter https://www.weisse-zone-rhoen.de/5g/offener-brief-andie-bundesregierung/.

4) Weitere Informationen, Stand 17.11.2020, abrufbar unter https:// ifurinstitut.wordpress.com/2020/04/24/corona-covid-19-symptoms-caused-by-60-ghz-frequencies/.

5) Nach einer Auswertung des Umweltbundesamtes liegt der Anteil der erneuerbaren Energien am Gesamtenergieverbrauch der Bundesrepublik im Jahr 2019 bei 17,1 \%; betrachtet man nur die Stromproduktion liegt der Anteil erneuerbarer Energien im Jahr 2019 bei 42,1\%; Umweltbundesamt, Erneuerbare Energien in Deutschland - Daten zur Entwicklung im Jahr 2019, Stand 17. 11.2020, abrufbar unter https://www.umweltbundesamt.de/sites/default/files/medien/1410/publikationen/2020-04-03_hgp-ee-in-zahlen_bf.pdf. 
Nicht umsonst weisen auch die Vereinten Nationen auf den direkten Zusammenhang von 5G-Ausbau und Umweltschutz hin. Schon heute liegt das Internet beim weltweiten Stromverbrauch auf Platz 3, ist also mitverantwortlich für den Klimawandel. ${ }^{6}$

Bisherige Untersuchungen nähren zumindest den begründeten Verdacht schädlicher Auswirkungen der Funkstrahlung. Konkret lassen sich eine Beeinflussung der Durchblutung des Gehirns, eine Beeinträchtigung der Spermienqualität, eine Destabilisierung der Erbinformation sowie Auswirkungen auf die Expression von Genen, nämlich den programmierten Zelltod und oxidativen Zellstress, daraus ableiten. $\mathrm{Ob}$ das $\mathrm{zu}$ realen Gesundheitsgefährdungen führt, bedarf noch weitergehender Untersuchungen.

Aus Vorsorgegründen haben Städte wie Brüssel, Florenz, die Kantone Genf und Jura den 5G-Ausbau zunächst gestoppt und zwar so lange bis die gesundheitliche Unbedenklichkeit der Funkstrahlung wissenschaftlich nachgewiesen ist.

$\mathrm{Zu}$ dem Themenfeld existieren weltweit unzählige Forschungsberichte mit unterschiedlichen Ergebnissen. Diese lassen erkennen, dass die Experten bis heute zu keiner einheitlichen und eindeutigen Aussage darüber gelangt sind, ob und welche gesundheitlichen Auswirkungen der Ausbau von $5 \mathrm{G}$ für den Menschen haben wird. Gewisse Popularität hat die Ramazzini-Studie des US-Departments of health and human servicies aus März $2018^{7}$ erlangt, wo die krebserzeugende Wirkung schon der 3G- und 4G-Funstrahlung bei Feldstärken unterhalb der Grenzwerte nachgewiesen wurde. Die Branche der Mobilfunkbranche und der von diesen majorisierte ICNIRP hat zwar sofort eine Gegendarstellung dazu veröffentlicht, die aber schon bei bloßer Lektüre als ausgesprochen schwach gewertet werden kann. ${ }^{8}$ Der reine Wirkmechanismus scheint dagegen geklärt: Durch die Funkstrahlen werden die Wasserstoffbrücken der „Stöpsel“ in den Kalzium-Kanälen der Zellwände geöffnet; daher strömt ständig Kalzium ein, das eine Reihe chemischer Reaktionen auslöst und zur Bildung Freier Radikale führt, die die DNA angreifen. So kommt es zum Anstieg typischer sehr aggressiver Tumore im Gehirn (Glioblastome im Frontal- und Seitenlappen), die weltweit stark zunehmen.

Aktuell und informativ ist der Endbericht des Instituts für Technikfolgen-Abschätzung der Österreichischen Akademie der Wissenschaften aus Januar 2020. Es handelt sich dabei zwar nicht um eine wissenschaftliche Studie, sondern eher um einen politischen Kompromiss, der aber gut die Dichotomie des Themas aufzeigt. In dem im Auftrag des Österreichischen Parlaments erarbeiteten Endbericht zum Titel ,5G-Mobilfunk und Gesundheit" geht es um eine aktuelle Einschätzung des Evidenzstandes zu möglichen Gesundheitsrisiken von elektromagnetischen Feldern des Mobilfunks durch anerkannte wissenschaftliche Gremien. Der Bericht kommt nach einer Auswertung nationaler und internationaler Stellungnahmen maßgeblicher internationaler wie auch nationaler wissenschaftlicher Gremien im Hinblick auf den Evidenzstand der einzelnen Äußerungen zu keinem eindeutigen Ergebnis. In der Zusammenfassung dieser aktuellen Untersuchung heißt es:

„Vor diesem Hintergrund ist keine eindeutige und konsensuale Feststellung des gesundheitlichen Risikopotenzials bezüglich etablierter Mobilfunkstrahlung absehbar. In Bezug auf $5 \mathrm{G}$ im Speziellen scheint der Mangel an tierexperimentellen und In-vitro-Studien prinzipiell behebbar. Die Produktion einer robusteren Evidenzsituation ist jedoch nicht absehbar (anspruchsvolle Studiendesigns) bzw. unwahrscheinlich (Fehlen von epidemiologischen Studien).“

Daraus werden Empfehlungen abgeleitet wie

- eine umsichtige Vermeidung der Strahlung,

- der Formulierung spezifischer Leitlinien für Sendeanlagen, für das technische Design von Endgeräten und die Gestaltung der Grundversorgungsinfrastruktur,
- weitere unabhängige, nationale und internationale Forschung in höchster Qualität zur Gewährleistung möglichst robuster Evidenz,

- größere Klarheit und gezieltere Kommunikation zu technologischen Details aller geplanten Ausbaustufen, tatsächlich erwartbaren Anwendungsbereichen und Expositionssituation,

- neue Wege der Unsicherheits- und Risikoinformation vor dem Hintergrund einer nicht endgültig auflösbaren Unsicherheit, Widersprüchlichkeit und Kontroversialität,

- neue Wege und Orte des Unsicherheits- und Risikodialogs vor dem Hintergrund des hohen Bedarfs an Austausch von Seiten unterschiedlicher Stakeholder und dem Fehlen unabhängiger Institutionen für die Organisation und Moderation eines solchen inter- und transdisziplinären Austausches in Österreich sowie

- der Evaluierung des Risiko-Governance-Systems, z.B. im Hinblick auf die institutionelle Trennung und Legitimationskriterien von Bewertung, Empfehlung, politischer Entscheidung und Management.

$\mathrm{Zu}$ verweisen ist auch darauf, dass die Rückversicherungen die 5G-Technologie als Hochrisikotechnologie einstufen und Versicherungsschutz ablehnen. Die Mobilfunkbetreiber können sich also nicht gegen die Mobilfunkrisiken versichern.

In juristischer Hinsicht stellt sich vor dem Hintergrund der diffusen naturwissenschaftlichen Ausgangslage die Frage, wie der Ausbau des 5G-Netzes geplant, moderiert und schlussendlich gestaltet werden kann mit dem Ziel, möglichst großen Nutzen zu generieren und möglichst geringen Schaden zu erzeugen. Da der Ausbau weltweit vonstattengehen wird, sollten solche Erkenntnisse am besten weltweit genutzt werden können, zumindest auf EU-Ebene.

\section{Der europäische Gesundheitsschutz}

Der Schutz der Gesundheit und des menschlichen Lebens ist im europäischen Recht von hohem Rang (Artt. 3, 4, 5, 35 GRCh). So ist gemäß Art. 168 Abs. 1 S. 1 AEUV bei der Festlegung und Durchführung aller Unionspolitiken und Maßnahmen ein hohes Gesundheitsschutzniveau sicherzustellen. Art. 168 AEUV begründet ausdrücklich gesundheitspolitische Kompetenzen der Union. Dazu gehört die Verbesserung der Gesundheit der Bevölkerung, die Verhütung von Krankheiten, die Erforschung der Ursachen und deren Beobachtung, die frühzeitige Meldung und die Bekämpfung schwerwiegender grenzüberschreitender Gesundheitsgefahren in den Kompetenzbereichen der Union. Die Kommission kann dazu ausdrücklich Initiativen ergreifen, die darauf abzielen, Leitlinien Indikatoren festzulegen und zum Bestandteil andere Politiken zu machen.

Art. 36 AEUV erlaubt Einfuhr-, Ausfuhr- und Durchfahrverbote und -beschränkungen unter anderem zum Schutze der Gesundheit und des Lebens von Menschen. Nach der Cassis-Rechtsprechung des $\mathrm{EuGH}^{9}$ stellt der Gesundheitsschutz zudem auch einen ungeschriebenen Rechtfertigungsgrund für Einfuhr-, Ausfuhr- und Durchfuhrverbote und -beschränkungen dar. ${ }^{10}$

6) Thiede, Internet und Mobilfunk sind Feinde des Klimas!, in: Die Welt v. 23.8.2019.

7) Stand 17.11.2020, abrufbar unter https://www.umweltbundesamt.de/sites/default/files/medien/1410/publikationen/2020 04-03_hgp-ee-in-zahlen_bf.pdf.

8) Stand 17.11.2020, abrufbar unter https://pubmed.ncbi.nlm.nih. gov/31464775/.

9) EuGH, Urt. v. 20.2.1979 - 120/78, BeckRS 9998, 104243 (Rdnr. 8).

10) Frenz, Handbuch des Europarechts Bd. 1, 2. Aufl. 2012, \5 Rdnr. 1161. 
Die hohe Bedeutung des Gesundheitsschutzes wird mit der Querschnittsklausel des Art. 9 AEUV und dem Ziel eines hohen Schutzniveaus nach Art. 168 Abs. 1 AEUV, das entsprechend Art. 168 Abs. 2 AEUV nicht ausschließlich durch die Union erreicht werden muss, noch gesteigert. ${ }^{11}$ Auf diesen Gesichtspunkt hat auch der EuGH immer wieder zurückgegriffen. ${ }^{12}$ Mitgliedstaaten dürfen im Einzelfall sogar ein höheres Schutzniveau vorsehen als gemeinschaftliche Harmonisierungsmaßnahmen, wenn sie nachweisen können, dass die Einzelmaßnahme ein höheres Niveau des Gesundheitsschutzes gewährleistet. ${ }^{13}$ Dabei hängt der Grad der Substantiierungspflicht als Ausprägung des Verhältnismäßigkeitsgrundsatzes von der Intensität der im Einzelfall drohenden Gefahr und ihrer Eintrittswahrscheinlichkeit ab. ${ }^{14}$

So hat der EuGH im Bereich der Zulassung von Lebensmitteln, Arzneimitteln und Nahrungsergänzungsmitteln wiederholt entschieden, dass, soweit beim gegenwärtigen Stand der wissenschaftlichen Forschung noch Unsicherheiten bestehen und solange es noch keine Harmonisierung auf EU-Ebene gibt, jeder einzelne Mitgliedstaat der Gemeinschaft zu bestimmen hat, in welchem Umfang der Schutz der Gesundheit und des Lebens von Menschen gewährleistet werden soll und ob es für die Zulassung der Mittel einer vorherigen Zulassung bedarf. ${ }^{15}$ Die Entscheidungen betonen die besondere Bedeutung des Gesundheitsschutzes, hier konkret vor der Einnahme von Mitteln, die bei zu hoher Dosierung eine schädliche Wirkung hervorrufen können. ${ }^{16}$ Dabei sind die Mitgliedstaaten auf den Grundsatz der Verhältnismäßigkeit verpflichtet und müssen die gewählten Maßnahmen auf das Maß beschränken, das zum Schutz der Gesundheit der Bevölkerung tatsächlich erforderlich ist.

Um dem individuellen Gesundheitsschutz zur Geltung $\mathrm{zu}$ verhelfen, wurde im Bereich der Luftreinhaltung die Betroffenheit und damit die Klagebefugnis eines einzelnen nach Art. 267 AEUV bereits dann anerkannt, wenn eine Grenzwertüberschreitung an sich noch keine Gesundheitsgefährdung bedeutet, sondern die zugrunde liegende Vorschrift die menschliche Gesundheit als Bestandteil des Umweltschutzes mit schützt. ${ }^{17}$

Auch im Bereich der Umweltpolitik steht der Schutz der menschlichen Gesundheit an oberster Stelle. So besagt der Grundsatz 1 der Rio-Erklärung über Umwelt und Entwicklung von 1992, dass die Menschen im Mittelpunkt der Bemühungen um eine nachhaltige Entwicklung stehen und dass sie ein Recht auf ein gesundes und produktives Leben im Einklang mit der Natur haben.

Daraus kann die Schlussfolgerung gezogen werden, dass dem europäischen Verordnungs- und Richtliniengeber die Gesundheitsgefährdungen, die durch Mobilfunkstrahlung ausgelöst werden könnten, nicht gleichgültig sein dürfen. Er muss sich mit ihnen näher befassen.

Dies entspricht auch einer Empfehlung des Rates vom 12.7.1999 zur Begrenzung der Exposition der Bevölkerung gegenüber elektromagnetischen Feldern. ${ }^{18}$ Schon 1999 wurde es als unbedingt notwendig erkannt, die Bevölkerung vor nachweislich gesundheitsschädlichen Auswirkungen zu schützen, die als Folge der Exposition gegenüber elektromagnetischen Feldern eintreten können. Dazu empfiehlt der Rat, nach sorgfältiger Auswertung aller veröffentlichten wissenschaftlichen Literatur ein System von Basisgrenzwerten und Referenzwerten einzuführen, um daraus Immissionsgrenzwerte abzuleiten und ein hohes Schutzniveau für die Bürger zu gewährleisten. Der Rat fordert die Kommission unter anderem auch dazu auf, die Erforschung der lang- und kurzfristigen Auswirkungen einer Exposition gegenüber elektromagnetischen Feldern in allen relevanten Frequenzbereichen durch Forschungsprogramme zu fördern und auf einen internationalen Konsens im Hinblick auf Leitlinien und Empfehlungen für Schutzund Vorsorgemaßnahmen hinzuwirken.

\section{Das Vorsorgeprinzip}

Angesichts der naturwissenschaftlichen Erkenntnisdefizite über die möglichen Folgen der Mobilfunkstrahlung liefert auch das Vorsorgeprinzip - oder auch Vorsorgegrundsatz genannt - wichtige Hinweise auf die zu erfüllenden Notwendigkeiten vor der Einführung einer neuen Technik. Das Vorsorgeprinzip ist nicht nur national in Art. 20a GG verankert, sondern zudem auch EU-rechtlich und völkerrechtlich vorgegeben. Auch neue technologische Entwicklungen unterliegen dem Vorsorgeprinzip.

\subsection{Der Vorsorgegrundsatz aus Art. 191 Abs. 2 S. 1 AEUV}

Gemäß Art. 191 Abs. 2 S. 2 AEUV beruht die Umweltpolitik der EU auf den Grundsätzen der Vorsorge und der Vorbeugung.

Der Vorsorgegrundsatz spielt auch in vielen Entscheidungen des EuGH eine wichtige Rolle, wozu nachfolgend einige ausgewählte Beispielsfälle genannt werden.

- Muss eine Behörde einen Plan oder ein Projekt genehmigen und bestehen Unsicherheiten darüber, ob der Plan oder das Projekt nachteilige Auswirkungen auf ein Habitatschutzgebiet aufweist, dann verpflichtet der EuGH die Behörde zur Anwendung des in Art. 6 Abs. 3 S. 2 der FFH-Richtlinie 92/43 EWG ${ }^{19}$ vorgesehene Vorsorgeprinzip anzuwenden und die durch den Plan oder das Projekt entstehende Beeinträchtigung der Schutzgebiete wirksam zu verhindern. ${ }^{20}$

- Auch bei der Zulassung von Projekten wie der Errichtung eines Kraftwerks muss die Genehmigungsbehörde im Rahmen der Durchführung der FFHVerträglichkeitsprüfung nach dem Vorsorgegrundsatz die Schutzmaßnahmen berücksichtigen, mit denen etwaige unmittelbar verursachte schädliche Auswirkungen verhindert oder verringert werden sollen, um dafür zu sorgen, dass das Projekt das geschützte Gebiet nicht beeinträchtigt. ${ }^{21}$

- Dasselbe gilt, soweit über Ausnahmen nach Art. 16 Abs. 1 FFH-Richtlinie zu entscheiden ist. Ergibt die Prüfung der besten verfügbaren wissenschaftlichen Daten eine Ungewissheit darüber, ob der günstige Erhaltungszustand einer Population einer vom Aussterben bedrohten Art trotz der Ausnahmeregelung gewahrt oder wiederhergestellt werden kann, dann sind

11) Frenz, Handbuch des Europarechts Bd. 1, 2. Aufl. 2012, \5 Rdnr. 1093.

12) EuGH, Rs. 104/75, Slg. 19 76,613 (Rdnr. 14/14)

13) EuGH, Urteil v. 20.3.2003 - C-3/00, EuZW 2003, $334 \mathrm{mit}$ Anm. Gundel $=$ BeckRS 2004, 76132 (Rdnr. 64)

14) Kingreen, in: Callies/Ruffert, EUV AEUV, 4. Aufl. 2011, Art. 34-36 AEUV, Rd. 199

15) EuGH, Urt. v. 14.7.1983-174/82, NJW 1984, 2757; EuGH, Urt v. 23.9.2003 - C-192/01. BeckRS 2004, 74834 (Kommission Dänemark), Rdnr. 42ff.; EuGH, Urt. v. 19.4.2004 - C-150/00, BeckRS 2004, 74457, Rdnr. 60; EuGH, Urt. v. 5.2.2004 C-24/00, BeckRS 2004, 75347 (Kommission/Frankreich), Rdnr. 50 ff.; EuGH, Urt. v. 5.3.2009 - C-88/07, BeckRS 2009, 70262, Rdnr. 86.

16) Streinz, Europarecht, JUS 2004, 905, 907

17) EuGH, Urt. v. 20.7.2008 - C-237/07, NuR 2008, 630; EuGH, Urt. v. 19.11.2014 - C-404/13, NuR 2015, 34 (Client Earth); EuGH, Urt. v. 3. 10.2019 - C-197/18, NVwZ 2019, 1587 mit Anm. Reinhardt $=$ ZUR 2019, 676 mit Anm. Henn = NuR 2019, 751.

18) ABl. 1999 L 199, S $59 \mathrm{ff}$

19) Richtlinie 92/43 EWG des Rates vom 21.5.1992 zur Erhaltung der natürlichen Lebensräume sowie wildlebenden Tiere und Pflanzen, ABl. 1992 L 206, S. 7 ff.

20) EuGH, Urt. v. 11. 4.2013 - C-258/11, NuR 2013, 343; EuGH, Urt. v. 15. 5. 2014 - C-521/12, Rdnr. 26-28, NuR 2014, 487.

21) EuGH, Urt. v. 26.4.2017 - C-142/16, NuR 2017, 393 Rdnr. 34 und 40 (Kraftwerk Moorburg) 
die Mitgliedstaaten nach dem in Art. 191 Abs. 2 AEUV verankerten Vorsorgegrundsatz dazu verpflichtet, von dem Erlass oder der Durchführung einer solchen Ausnahmeregelung abzusehen. ${ }^{22}$

- Auch bei der Prüfung von Plänen und Programmen besteht eine Verpflichtung, eine Verträglichkeitsprüfung durchzuführen, bereits dann, wenn anhand objektiver Umstände nicht ausgeschlossen werden kann, dass der entsprechende Plan oder das Projekt das Schutzgebiet erheblich beeinträchtig. ${ }^{23}$

- Auch beim Inverkehrbringen von Arzneimitteln rechtfertigt der Vorsorgegrundsatz den Erlass beschränkender Maßnahmen, wenn es sich als unmöglich erweist, das Bestehen oder den Umfang von behaupteten Risiken mit Sicherheit festzustellen. ${ }^{24}$ Der Generalanwalt stützt sich dabei in Rz. 139 auf das Urteil des EuGH im Fall Monsanto ${ }^{25}$, wo festgestellt wurde, dass Schutzmaßnahmen nicht mit einer rein hypothetischen Betrachtung des Risikos begründet werden können mit der Ausnahme der Bewertung von neuartigen Lebensmitteln.

- In einem Vorabentscheidungsersuchen zur Gültigkeit der Verordnung (EG) Nr. 1107/2009 des Europäischen Parlaments und des Rates vom ein 20.10.2009 über das Inverkehrbringen von Pflanzenschutzmitteln und zur Aufhebung der Richtlinie 79/117/EWG und 91/414/ $\mathrm{EWG}^{26}$ hat der EuGH ausgeführt, dass bei Unsicherheiten hinsichtlich des Vorliegens oder des Umfangs von Risiken für die menschliche Gesundheit Schutzmaßnahmen getroffen werden können, ohne dass abgewartet werden müsse, dass das Bestehen und die Schwere dieser Risiken vollständig dargelegt werden. ${ }^{27}$ Auf der anderen Seite verlangt der Vorsorgegrundsatz nur dann nach vorbeugenden Maßnahmen, wenn das Risiko, ohne dass seine Existenz und sein Umfang durch zwingende wissenschaftliche Daten in vollem Umfang nachgewiesen worden sind, auf der Grundlage der zum Zeitpunkt des Erlasses der Maßnahme verfügbaren wissenschaftlichen Daten hinreichend dokumentiert erscheint. ${ }^{28}$ Auf rein hypothetische Betrachtungen eines Risikos dürfen vorbeugende Maßnahmen dagegen noch nicht geschützt werden.

Im Ergebnis dürfen also Maßnahmen nicht auf rein hypothetischen Risiken beruhen, es muss andererseits aber auch nicht abgewartet werden, bis die Gefahr mit Sicherheit feststeht, da häufig absolute Gewissheit erst dann besteht, wenn sich das Risiko bereits materiell niedergeschlagen hat und es damit für Maßnahmen zur Gefahrenabwendung zu spät ist. ${ }^{29}$

Aus dieser Rechtsprechung folgt, dass dann, wenn ein gewisses Risiko für $5 \mathrm{G}$ auf der Grundlage wissenschaftlicher Daten hinreichend dokumentiert erscheint, was angesichts der Fülle der kritischen wissenschaftlichen Berichte der Fall ist, vorbeugende Maßnahmen zumindest getroffen werden können. Das Vorsorgeprinzip ist letztlich eine spezielle Ausprägung des Grundsatzes der Verhältnismäßigkeit. Hiernach sind die von der Wissenschaft offengelegten Risiken mit den konkreten Vorteilen, die mit $5 \mathrm{G}$ auch verbunden sind, abzuwägen. ${ }^{30} \mathrm{Je}$ schwerwiegender die gesundheitlichen Folgen für Teile der Bevölkerung ausfallen, umso stärkerer Restriktionen bedarf die Einführung dieser neuen Technologie.

\subsection{Der völkerrechtliche Vorsorgegrundsatz aus der Rio-Erklärung}

$\mathrm{Zu}$ erwähnen ist auch das Völkerrecht. Grundsatz 15 der Rio-Erklärung über Umwelt und Entwicklung von $1992^{31}$ fordert die Staaten dazu auf, zum Schutz der Umwelt im Rahmen ihrer Möglichkeiten allgemein den Vorsorgegrundsatz anzuwenden. Es heißt dort in Satz 2 wörtlich: „Drohen schwerwiegende oder bleibende Schäden, so darf ein Mangel an vollständiger wissenschaftlicher Gewissheit kein Grund dafür sein, kostenwirksame Maßnahmen zur Vermeidung von
Umweltverschlechterungen aufzuschieben“. Das völkerrechtliche Vorsorgeprinzip bildet das Leitbild des modernen Umweltvölkerrechts, wenngleich im Einzelfall schwer zu ermitteln ist, welches vorsorgende Handeln völkerrechtlich geboten ist. ${ }^{32}$ Es lassen sich diesem Grundsatz aber Leitlinien für den Umgang mit Unsicherheiten entnehmen, wie sie im Fall von $5 \mathrm{G}$ offensichtlich gegeben sind.

\subsection{Stellungnahmen der ICNIRP}

Ein gerne gebrauchtes Argument der Befürworter des Ausbaus von $5 \mathrm{G}$ liegt in einem Verweis auf Stellungnahmen der Internationalen Strahlenschutzkommission (ICNIRP), die im März 2020 das Schutzkonzept für elektromagnetische Felder auch für den neuen Mobilfunkstandard $5 \mathrm{G}$ bestätigt habe, sodass $5 \mathrm{G}$ unter Einhaltung der gesetzlichen Grenzwerte sicher betrieben werden könne. Die ICNIRP ist aber keine offizielle Einrichtung der EU, sondern eine in München ansässige und als privater Verein eingetragene Organisation, die von der Weltgesundheitsorganisation (WHO) und der Internationalen Arbeitsorganisation (ILO) formell als nichtstaatlicher Akteur und offizieller Partner anerkannt ist. Sie steht der Europäischen Kommission beratend zur Seite. Investigative Journalisten der Tageszeitung „Der Tagesspiegel“ haben im Januar 2019 die ICNIRP als „ein Kartell“ beschrieben, das systematisch alle Studien, die mögliche Gefahren für die menschliche Gesundheit belegen, entkräftet. ${ }^{33}$ Es wurde offengelegt, dass deren Mitglieder gleichzeitig in allen relevanten Institutionen aktiv sind und somit den offiziellen Diskurs kontrollieren. Die ICNIRP unterliegt selber keinen Kontrollen, sondern kontrolliert sich selbst und hält abweichende Meinungen fern. So ist es wohl auch kein Zufall, dass der Sitz der ICNIRP im Gebäude des Bundesamtes für Strahlenschutz in München beheimatet ist. Eine ausführliche Studie hat sich mit den beruflichen Hintergründen der Mitglieder der ICNIRP befasst und so ein einseitiges Gewicht der Mobilfunkbranche offengelegt. Von einem solchen Verband sind kaum objektive Stellungnahmen zu erwarten. ${ }^{34}$

So hat das Appellationsgericht (Berufungsgericht) $\mathrm{im}$ italienischen Bergamo mit Urteil vom 22.12.2009 (Az. 614/09) das gegenteilige Urteil des Landgerichts Brescia vom 15.5.2008 aufgehoben und dem Kläger wegen

22) EuGH, Urt. v. 10.10.2019 - C 674/17, NuR 2019, 756 Rdnr. 66.

23) EuGH, Urt. v. 7.9.2004 - C-127/02, Slg. I 2004, 7449; EuGH, Urt. v. 17. 4. 2018 - C- 441/17, NuR 2018, 327.

24) EuGH, Schlussantrag des Generalanwalts Paolo Mengozzi v. 4. 10.2018, BeckRS 2018, 23690, Rdnr. 140.

25) EuGH, Urt. v. 9.9.2003-C-236/01, BeckRS 2004, 75313.

26) ABl. 2009 L 309, S 1.

27) EuGH, Urt. v. 1.10.2019 - C-616/17, BeckRS 2019, 22830, Rdnr. 43

28) EuGH, Urt. v. 23.9. 2003 - C-192/01, BeckRS 2004, 74834, Rdnr. 100

29) EuGH, Urt. v. 23.9.2003 - C-192/01, BeckRS 2004, 74834, Rdnr. 101.

30) Sobotta, NuR 2020, 453, 456.

31) Vgl. „United Nations Conference on Environment and Development: Rio Declaration on Environment and Development", UN-Dok. A/CONF.151/26 (Vol. I); dazu: Hohmann, NVwZ 1993, $311 \mathrm{ff}$.

32) Durner, in: Landmann/Rohmer, Umweltrecht Bd. I, Stand: 92. Lfg. Febr. 2020, Umweltvölkerrecht, Rdnr. 63

33) Schumann/Simantke, Wie gesundheitsschädlich ist $5 \mathrm{G}$ wirklich?, in: Der Tagesspiegel v. 15.1.2019, S. 2.

34) Dazu ausführlich: Buchner/Rivasi, Die Internationale Kommission zum Schutz vor nichtionisierender Strahlung: Interessenkonflikte, „Corporate Capture“ und der Vorstoß zum Ausbau des 5G-Netzes, Juni 2020, Stand 17.11.2020, abrufbar unter https:// klaus-buchner.eu/die-internationale-kommission-zum-schutzvor-nichtionisierender-strahlung-interessenkonflikte-corporate-capture-und-der-vorstoss-zum-ausbau-des-5g-netzes/. 
eines Gehirntumors eine 80 \%ige Rente zugesprochen. In einem ähnlichen Fall hat das Appellationsgericht in Turin mit Urteil vom 3.12.2019 (Az. 904/2019) ebenfalls dem Kläger Recht gegeben und eine Entscheidung des Landgerichts Ivrea aus dem Jahr 2017 vollumfänglich bestätigt. Alle stattgebenden Urteile haben die Aussagen von Vertretern von ICNIRP ausdrücklich unberücksichtigt gelassen, weil diese parteiisch seien. Die Vorbehalte gegen die Stellungnahmen dieser Organisation scheinen also begründet zu sein.

\section{Folgerungen aus dem Vorsorgeprinzip und den Gesundheitsschutzanforderungen}

Es gibt in großer Anzahl ernstzunehmender Hinweise darauf, dass die intensive Strahlung gesundheitliche Risiken birgt und sogar Gesundheitsschäden verursachen kann. Es steht einem Juristen nicht zu, diese Untersuchungen inhaltlich zu bewerten. Allerdings macht die Zahl von annähernd 1500 Studien, die solche Gefährdungen zumindest in Teilen bestätigt haben, nachdenklich. Schon auf der Ebene der europäischen Normsetzung gibt das Veranlassung dazu, dem völkerrechtlichen Vorsorgeprinzip Genüge zu tun, also entweder den Mangel wissenschaftlicher Ungewissheit zu beseitigen oder kostenwirksame Maßnahmen zur Vermeidung von Umweltverschlechterungen zu ergreifen.

\subsection{Notwendigkeit einer Technikfolgenabschätzung}

Aus dem Schutzauftrag des europäischen Primärrechts und der Erkenntnis, dass gesundheitliche Risiken des verstärkten Mobilfunks $5 \mathrm{G}$ nicht ausgeschlossen werden können, muss die Folgerung abgeleitet werden, vor Einführung der Technik eine Technologiefolgenabschätzung durchzuführen. Diese ist vor allem ein Instrument der Politikberatung und dient der Vermittlung des Wissens zwischen Wissenschaft und Politik. Die Technologiefolgenabschätzung ersetzt keine Entscheidungen, sondern bereitet diese sachverständig vor. ${ }^{35}$ Erforderlich sind Untersuchungen, mit denen die potentiellen Auswirkungen der Mobilfunkstrahlung insbesondere im Hinblick auf den Schutz der menschlichen Gesundheit, aber auch der Umwelt (Tiere, Pflanzen, Luft usw.) näher untersucht wird.

\subsection{Ableitung eines Minimierungsgebotes}

Solange nicht überwiegende Erkenntnisse für die Ungefährlichkeit der Strahlung sprechen, muss zum Schutz der menschlichen Gesundheit, aber auch der Umwelt, ein Minimierungsgebot greifen. Die Strahlung ist also überall dort $\mathrm{zu}$ verhindern, wo sie verhindert werden kann und dort zuzulassen, wo sie einen überwiegenden Nutzen bringt.

Daraus kann weiter die Folge abgeleitet werden, dass $5 \mathrm{G}$ möglicherweise zur Steuerung von Produktionsprozessen und auch zur Steuerung des autonomen Fahrens von überragendem Nutzen ist und in diesen Bereichen auch zugelassen werden sollte. Keinesfalls ist damit aber gerechtfertigt, sämtliche Häuser und Wohnungen mit Funkstrahlen zu belasten und die sich dort aufhaltenden Personen zu gefährden. Denn dies hätte zur Folge, dass die Menschen und damit auch elektrosensible Personen der Strahlung nicht mehr ausweichen können. Gerade die eigene Wohnung muss als Rückzugsort und Schutzraum erhalten bleiben, was auch technisch möglich ist. Ein WLAN mit $5 \mathrm{G}$ ist dann überall dort zuzulassen, wo nicht Menschen schlafen und wohnen. Das bedeutet, dass eine entsprechende flächendeckende WLAN-Versorgung im öffentlichen Raum akzeptabel erscheint, ohne Zustimmung der jeweiligen Wohnungsnutzer aber nicht in privaten Wohnungen, in Wohnheimen, Krankenhäusern und dergleichen. ${ }^{36}$ Dort können die ent- sprechenden Signale auch per Kabel übertragen werden, sodass es jedem einzelnen Bewohner freigestellt ist, das Signal innerhalb seiner Wohnung kabellos, also durch WLAN, weiter zu übertragen. Hier muss dann durch technische Vorgaben sichergestellt werden, dass das WLAN-Signal nicht in Nachbarwohnungen eindringt, d.h. es sind Begrenzungen der maximalen Stärken vorzusehen. Kritsch zu sehen sind also einstweilen auch sog. Smart Meter, mit deren Hilfe Wohnungen einer hohen Strahlenbelastung ausgesetzt würden und das nur zu dem Zweck, den Versorgern von Strom, Gas und Wasser die jeweiligen Verbräuche zeitnah und permanent zu übermitteln. Dies hat zwar gewisse Vorteile, rechtfertigt aber die Strahlenbelastung der Bevölkerung so lange nicht, wie nicht die Ungefährlichkeit der $5 \mathrm{G}$-Strahlung nachgewiesen ist.

Durch eine solche, über die Wissenschaft vermittelte Zwischenlösung lassen sich die Vorteile von $5 \mathrm{G}$ dort nutzen, wo sie dringend gebraucht werden, und gleichzeitig weitgehend sicherstellen, dass die Gesundheit von Menschen nicht überproportional gefährdet wird, was einem berechtigten Schutzanliegen von Menschen entspricht. Vor allem elektrosensible Menschen sind weitestmöglich vor der starken Strahlenbelastung und damit vor Beeinträchtigungen ihres Wohlbefindens und ihrer Gesundheit zu schützen.

\subsection{Entwicklung neuer Begrenzungen/Grenzwerte}

Die Technologiefolgenabschätzung wird ergeben, bis zu welcher Strahlenbelastung gesundheitliche Schäden nicht ernsthaft zu befürchten sind und ab wo es zum Schutz der Gesundheit von Menschen und zum Schutz von Umweltgütern Begrenzungen geben muss.

Diese können dann auf EU-Ebene entweder durch eine neu zu schaffende EU-Verordnung, die unmittelbare Geltungskraft in allen EU-Mitgliedstatten hat, oder durch eine EU-Richtlinie, die die Mitgliedstaaten zur Umsetzung in das jeweilige nationale Recht verpflichtet, geschehen (Art. 288 Abs. 2 und 3 AEUV).

Unverständlich ist in diesem Kontext, dass die EU-Kommission am 30.6.2020 die Durchführungsverordnung für kleine drahtlose Zugangspunkte oder kleine Antennen verabschiedet hat, wonach kleinere 5G-Antennen europaweit genehmigungsfrei installiert werden dürfen mit der Zielrichtung, den Ausbau des 5G-Netzes zu beschleunigen. Hierbei wurden sämtliche Bedenken ausgeblendet, was als ein Ergebnis guter Lobbyarbeit der Mobilfunkbetreiber gewertet werden darf. Eine Technologiefolgenabschätzung ist unterblieben.

Auf nationaler Ebene können die Schutz- und Vorsorgewerte der 26. $\mathrm{BImSchV}^{37}$ entsprechend angepasst werden. Deren letzte Änderung stammt noch aus dem Jahr 2013 und berücksichtigt von daher noch nicht die zahlreichen neueren Forschungsergebnisse auf dem Fachgebiet der Strahlungsforschung. Denn die 26. BImSchV verfolgt ausdrücklich das Ziel, vor möglichen gesundheitlichen Beeinträchtigungen und Risiken durch elektrische, magnetische und elektromagnetische Felder zu schützen. ${ }^{38}$

35) Kloepfer, Umweltrecht, 4. Aufl. 2016, $\mathbb{1} 1$ Rdnr. 85.

36) Der EGMR hat entschieden, dass das Menschenrecht auf Achtung der Wohnung (Art. 8 Abs. 1 EMRK) durch die behördliche Genehmigung von Immissionen des Mobilfunks tangiert sein kann und somit einer gesetzlichen Rechtfertigung (Art. 8 Abs. 2 EMR K) bedarf, EGMR, Entsch. v. 3. 7.2007 - 32015/02, NVwZ 2008, 1214. An dieser gesetzlichen Normierung des Mobilfunks mit entsprechenden Grenzwerten auch zum Schutz vor den athermischen Wirkungen der Strahlung aber fehlt bis heute.

37) 26. Verordnung zur Durchführung der Bundes-Immissionsschutzgesetzes (Verordnung über elektromagnetische Felder 26. BImSchV) i. d. F. v. 14.8.2013, BGBl. I S. 3266.

38) BR-Drs. 209/13, S. 33. 